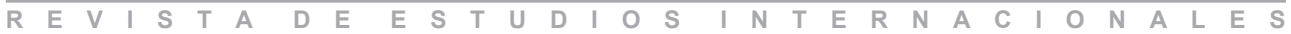 \\ La política antártica chilena: nuevas realidades
}

\author{
Maria Teresa Infante Caffi
}

\section{LAS DEFINICIONES}

1

Hacia dónde se encamina la política antártica de Chile?, ¿debe ella concentrarse en los temas de modernización institucional y de organización interna, o debe poner más acento en los desafíos del Sistema Antártico y su capacidad para responder a sus diversos ámbitos de acción?

El 2 de julio de 1906, el ministro de Relaciones Exteriores de Chile, Antonio Hunneus Gana, escribía al ministro de Marina y de Guerra de la época, diciendo que:

El Gobierno está animado del propósito de hacer efectiva por todos los medios prácticos a su alcance la Soberanía que inviste sobre las vastas Islas Australes i sobre el Continente Austral que hasta hoy permanecen aparentemente abandonados, consolidando así por medio de la ocupación sus títulos al dominio de la zona antártica ${ }^{1}$.

El mismo año, se creaba la primera Comisión Chilena Antártica a la que se le encomendó preparar:

debidamente una expedición a la antártica americana que verifique un reconocimiento de esas regiones y que haga en ellas investigaciones científicas, sobre todo magnéticas, haciendo así efectivos los incuestionables derechos de soberanía de Chile en ese sector ${ }^{2}$.

En esa época Chile otorgaba concesiones pesqueras a Pedro F. Benavides (1902) y a Toro y Fabry $(1906)^{3}$, y la Sociedad Ballenera de Magallanes era autorizada para operar en aguas antárticas, instalando una base en isla Decepción.

\footnotetext{
A. Hunneus Gana, Antártida, Santiago, Imprenta Chile, 1948.

Ibid.

Sobre el Decreto Supremo No 260 de 27 de febrero de 1906, que otorgara la concesión Fabry y de Toro Herrera, ver Pinochet, O., La Antártica Chilena, Santiago, Ed. del Pacífico, 1955 (3ª ed.). Para una exposición de la práctica chilena en esos años, ver Bush, W.M., Antarctica and International Law. Vol.II, Londres, Oceana Publications, Inc., 1982, pp. 289-299.
} 
Chile iniciaba intercambios diplomáticos con Argentina invitándola a delimitar los territorios antárticos, y a emprender cooperativamente la exploración en esas tierras ${ }^{4}$. Se hablaba de la Antártida americana $^{5}$, y el geógrafo Luis Risopatrón titulaba una obra precisamente de esa for$\mathrm{ma}^{6}$, definiendo como tal la parte de las tierras antárticas encerradas entre los meridianos extremos de la América Meridional, desde el grupo de las islas Sándwich del Sur, en la latitud $55^{\circ}$, hasta la isla Pedro I $\left(70^{\circ} \mathrm{S}\right)$.

\section{Chile inició intercambios diplomáticos} con Argentina invitándola a delimitar los territorios antárticos.

La ciencia, los emprendimientos económicos, las negociaciones diplomáticas y la consolidación de derechos en ese continente dieron contenido a una serie de definiciones políticas que se proyectaron más allá de la época. Jorge Berguño destaca que la declaración de los límites antárticos formulada por el Presidente Pedro Aguirre Cerda en 1940 recuerda el precedente de 1906, sumando a los enfoques geográficos e históricos el método del sector, adaptado a la Antártica ${ }^{7}$. En esos años, las preocupaciones diplomáticas globales se situaron en un ámbito más competitivo, y nuevamente Chile y Argentina sostuvieron conversaciones para cooperar en torno a una Antártica Sudamericana ${ }^{8}$.

La política antártica contemporánea tiene raíces profundas en las experiencias mencionadas, que aún se proyectan en los temas del presente y conservando rasgos vinculados al patrimonio político de la primera época del siglo XX. Además se relaciona con los emprendimientos balleneros, la posición geográfica de la región magallánica, y la apertura intelectual al conocimiento de las tierras polares, así como con reflexiones jurídicas acordes con los debates internacionales de la época.

Actualmente predomina otro ambiente internacional. También son otras las

4 Estas negociaciones no prosperaron, pero quedó un interesante proyecto de «Tratado complementario de demarcación de límites», elaborado por Chile y propuesto a Argentina. Texto en inglés en Beagle Channel Arbitration, Pleadings of Chile, 1973, Vol. II, p. 243. Citado por W.M. Busc, ibid., pp. 303-304. Referencias también en Pinochet, O., «Antecedentes históricos de la política internacional de Chile en la Antártica. Negociaciones chileno-argentinas de 1906, 1907 y 1908», en Orrego V., Francisco et al., Política Antártica Chilena, Santiago, Instituto de Estudios Internacionales de la Universidad de Chile, pp. 75-76.

5 En un mapa preparado por el Director de la Oficina de Límites, Luis Risopatrón, se representa este territorio con el nombre de Antártida Americana.

6 La Antártica Americana, publicado en Santiago por los Anales de la Universidad de Chile, Imprenta Cervantes, 1908.

7 Berguño, J., Cincuenta años de política antártica», Anales, Medio Siglo de Política Antártica (19401990), Santiago, 1991, pp. 26-27

8 Debe recordarse el aporte del general Ramón Cañas Montalva, quien dirigió la Revista Geográfica de Chile «Terra Australis». Entre otros trabajos, ver «Chile, el más antártico de los países del orbe y su responsabilidad continental en el Sur-Pacífico», Revista Geográfica de Chile, No 4, 1950, pp. 23-40, y «El valor geopolítico de la posición Antártica de Chile», Revista Geográfica de Chile, No 9, 1953, pp.11-16. 
preocupaciones y exigencias que se plantean a nuestro país en su relación con la Antártica. En el período 2007-2008 se celebrará el Año Polar Internacional ${ }^{9}$ y es cada vez más pertinente preguntar si el Estado cuenta con los medios -si no con las decisiones- para responder con la velocidad y la eficacia necesarias a un Sistema exigente en responsabilidades, a partir de definiciones que asumen el carácter que posee el país como reclamante de derechos soberanos en la Antártica.

\section{ElAño Geofísico Internacional 1957-1958 fue una de las bases directas del Tratado Antártico.}

El Año Polar Internacional es un acontecimiento de rango mundial que abarcará también a la Antártica como uno de los centros de atención principales. Este nuevo año polar es el siguiente después del Año Geofísico Internacional 19571958 que sirvió como una de las bases directas del Tratado Antártico, situando la libertad de investigación científica entre sus pilares y nervio del desarrollo del sistema ${ }^{10}$. El número de Estados participan- tes será esta vez mayor (31 contra 12 en el período anterior), con científicos de más de 60 nacionalidades, y los temas en que se centrará su atención serán las relaciones entre los sistemas terrestres, oceánicos y atmosféricos mundiales y los procesos que ocurren en las zonas polares, una de las cuales es la Antártica.

En lo inmediato, hace pocos meses culminó la XXIX Reunión Consultiva del Tratado Antártico ${ }^{11}$, donde fueron claramente puestos de relieve los temas de la cooperación científica y logística como uno de los supuestos de la actividad antártica, junto con el aporte de las regiones polares al estudio del cambio climático, la cuestión del turismo antártico y las relaciones con el Consejo Ártico, que agrupa a los países vecinos a ese océano.

En su entorno internacional, la política contemporánea se estructura a partir del supuesto de que existe un Sistema Antártico $^{12}$, creado desde que emerge del Tratado de Washington de 1959 y que se ha fortalecido con el tiempo, todo lo cual implica también una forma de asociación internacional entre una pluralidad de Estados abierta a las influencias de los procesos globales, especialmente ambienta-

9 El Año Polar Internacional será patrocinado por el Consejo Internacional para la Ciencia (CIUS) y la Organización Meteorológica Internacional (OMM). Los años polares anteriores tuvieron lugar en 1882-1883, 1932-1934 у 1957-1958

10 León C., et al., La Antártica y el Año Geofísico Internacional. Percepciones desde Fuentes Chilenas. 1954-1958, Valparaíso, Universidad de Playa Ancha, 2006

11 Celebrada entre el 12 y el 23 de junio de 2006. La XXIX Reunión Consultiva del Tratado Antártico aprobó la Declaración Antártica de Edimburgo sobre el Año Polar Internacional 2007-2008, $<$ www.atcm206.gov.uk>.

12 El Protocolo al Tratado Antártico sobre Protección del Medio Ambiente, de 1991, indica que el Sistema significa el Tratado Antártico, las medidas en vigor según ese Tratado, sus instrumentos internacionales conexos que se encuentran en vigor y las medidas en vigor de acuerdo con esos instrumentos (Artículo, 1 (e)). 
les ${ }^{13}$, donde se mantienen las bases del arreglo jurídico-político respecto del territorio establecido por el artículo IV del Tratado. A la generación del contenido de este Artículo, el statu quo territorial, con indicaciones claras sobre los sujetos a que se refiere su texto, de alcance un tanto ambivalente, contribuyó en forma decisiva nuestro país, tomando en cuenta los trabajos encomendados por el gobierno al profesor Julio Escudero Guzmán ${ }^{14}$. Esta definición no ha sido objetada o modificada posteriormente.

\section{El ámbito en que se desarrolla la política antártica influye en materias políticas, jurídicas y presupuestarias.}

\section{LAS INSTITUCIONES}

Junto a este entorno, la política antártica también se desenvuelve en un ámbito institucional y burocrático interno, que influye y decide en materias políticas, jurídicas y presupuestarias, así como respecto de las inversiones en infraestructura, la programación de las investigaciones y el desarrollo de reglamentaciones naciona- les. Sus fortalezas y la capacidad para conducir las decisiones y servir de base para coordinar posiciones entre diversos sectores se ponen a prueba en forma periódica, a lo menos cada año, con ocasión de las discusiones presupuestarias y cuando se prepara la participación nacional en la Reunión Consultiva del Tratado Antártico, también anual.

De igual manera lo son las acciones emprendidas por el Estado o los organismos que actúan bajo su alero en cuanto a su capacidad para poner en sintonía los objetivos sectoriales con los grandes temas internacionales que influyen en el debate propio del Sistema Antártico. El juicio sobre tales capacidades lo hacen en un caso los actores internos, estatales y no estatales ${ }^{15}$, y en el otro, gobiernos extranjeros y entidades internacionales, gubernamentales y no gubernamentales.

Para enfrentar el tema de la política antártica se puede optar entonces por un enfoque que privilegie el estudio de los mecanismos institucionales y profundice lo relativo a las cuestiones atinentes a los organismos que la formulan o ejecutan, sus carencias y atribuciones, y la forma de resolver las cuestiones relacionadas con la jurisdicción en la Antártica, o por un aná-

13 Por ejemplo, la Declaración de Edimburgo de 2006 destaca que el Año Polar Internacional es una iniciativa conjunta de la Organización Meteorológica Mundial y del Consejo Internacional para la Ciencia, para un mejor conocimiento de los sistemas terrestres, oceánicos y atmosféricos que controlan el planeta.

14 Gajardo Villarroel, E., «Antecedentes de la Negociación Diplomática previa al Tratado de 1959 y la posición de Chile», en Orrego Vicuña, F., Infante Caffi, M. T. y Armanet Armanet, Pilar, Política Antártica de Chile, Santiago, Instituto de Estudios Internacionales, Universidad de Chile, 1984, pp. 81-89.

15 El Programa Consejo de Política Antártica, aprobado en 2003, fue evaluado desde esa perspectiva por la Dirección de Presupuestos en 2004. Informe Final de Evaluación, junio 2004, <www.dipres.cl/ docs/EPF060400072004_01>. 
lisis que tome en consideración los grandes asuntos que definen el debate internacional antártico, considerando sus implicaciones para el desarrollo o el afianzamiento de posiciones nacionales, y la proyección e impacto de los programas.

\section{El Comité para la Protección del Medio Ambiente tiene vastas consecuencias para la realización de actividades en la Antártica.}

Diversos análisis internos se han referido a esos temas, inclinándose por una u otra posición ${ }^{16}$. En todos los casos, el papel de las instituciones diplomáticas, en especial del Ministerio de Relaciones Exteriores, del Instituto Antártico Chileno que forma parte del mismo, de las Fuerzas Armadas y del Ministerio de Defensa, de la XII Región de Magallanes y la Antártica Chilena $^{17}$, y en lo que fuere pertinente de la Dirección Nacional de Fronteras y Límites de la Cancillería, de la comunidad académica y el entorno no gubernamental y su inserción en el marco oficial, son elementos importantes. Esto último se plantea tanto en torno a la ciencia como respecto de los enfoques que visualizan a la Antártica como un medio para realizar actividades con contenido económico, aunque sean altamente reguladas internacionalmente.

Esta situación se hace particularmente evidente en cuanto a la aplicación del Protocolo al Tratado Antártico sobre Protección del Medio Ambiente de 1991 (Protocolo de Madrid), vigente desde 1998, que establece un Comité para la Protección del Medio Ambiente, y que como instrumento postula un mayor compromiso respecto de la protección del medio ambiente y los ecosistemas dependientes y asociados, y el valor intrínseco de la Antártica tiene vastas consecuencias para la planificación y realización de actividades en esa región. Su aplicación requiere dotarse de mecanismos e instancias aptos para evaluar el impacto ambiental y conjugar los mecanismos vigentes en general en el país con las características propias de un régimen con participación de otros Estados.

ACTUALIDAD DE LA POLÍTICA.

LAS EXIGENCIAS DE LA MODERNIZACIÓN DEL ESTADO

La versión oficial más actual de la política antártica chilena data de 2000 , cuando se promulgó el texto aprobado por

16 A manera de ejemplo cabe citar la serie de seminarios organizados por el Consejo de Defensa del Estado en 2005, en Puerto Williams, Punta Arenas y Santiago. En ellos se examinaron temas como la jurisdicción, la protección del medio ambiente y la institucionaldidad antártica. Las presentaciones permanecen inéditas.

17 El Estatuto Antártico de 1956 (DF Ley No 298, de 1956) atribuye al Intendente de Magallanes el conocimiento y resolución de todos los asuntos administrativos referentes a la antártica chilena, norma que ha sido superada por el crecimiento del Sistema Antártico y las tareas que incumben al Gobierno chileno y en especial a su Cancillería. 
el Consejo de Política Antártica en $1999^{18}$. La Política prioriza las siguientes áreas:

- La participación efectiva en el Sistema del Tratado Antártico,

- El fortalecimiento de la institucionalidad antártica nacional,

- La preservación de la paz, actividades científicas y reserva ecológica, - La cooperación internacional,

- La promoción de facilidades en Chile continental como «país-puente» a la Antártica, fortaleciendo la participación de la Región de Magallanes y Antártica Chilena de acuerdo con las competencias administrativas que le otorga la legislación nacional,

- La orientación de la ciencia nacional antártica de forma que se vincule a las grandes tendencias,

- La conservación de los recursos vivos marinos y la pesca austral, y

- La promoción del turismo controlado.

Esta política, que a nuestro juicio está vigente, sugiere que Chile debe adoptar un enfoque multidimensional para el tratamiento de los asuntos antárticos. Para ello, asume la existencia de un sistema del cual es parte, a cuyo fortalecimiento y legitimación internacional ha contribuido, $\mathrm{y}$ ese contexto es el que entrega las bases para una perspectiva científica nacional antártica y otras decisiones de alcance general.

Los sucesivos gobiernos han recono- cido el papel no solo de orientación de las grandes tendencias en materia científica antártica, sino también que se trata de un antecedente fundamental para definir las medidas de incentivo que el país debería tomar en materia científica, su financiamiento y su proyección en el plano interno. Desde el punto de vista de las medidas que implican una asignación presupuestaria para la Antártica, se trata de un tema altamente relevante.

\section{Dos límites ordenan el ámbito de acción de la política antártica.}

De este documento derivan exigencias para que la acción política muestre un alto grado de coordinación nacional real y efectiva, y también para que se compatibilice la acción privada, incluyendo la empresarial interesada en los recursos naturales (esencialmente los recursos vivos marinos antárticos) y el turismo, con enfoques sustentables y armónicos con las normas internacionales. Es decir, se trata de un documento que no sitúa la cuestión antártica privilegiando el nivel de los principios o que obligue a obtener resultados normativos a nivel internacional.

Dos límites ordenan el ámbito de acción de la política. Por una parte la necesidad de contar con una dirección superior para la coordinación de los organismos del Estado que poseen competencias en asuntos antárticos, donde se espera

18 El documento había sido preparado en 1998-1999 por el Instituto Antártico Chileno y la Dirección Nacional de Fronteras y Límites de la Cancillería, pero fue promulgado en el gobierno entrante en 2000. El embajador Jorge Berguño fue la persona que más influyó en su preparación. 
que el Ministerio de Relaciones Exteriores -que cuenta con una Dirección a cargo de los temas antárticos (DIMA) y del Instituto Antártico Chileno- cumpla con la misión de conjugar los intereses fundamentales y los sectores que los proyectan, y por la otra, la necesidad de determinar periódicamente prioridades efectivas, atribuyendo responsabilidades para llevar a cabo acciones que trasciendan el ámbito nacional en materia científica y que sean capaces de integrar otras actividades organizadas, como el turismo y la interrelación de la Antártica con el desarrollo de Magallanes.

Esto requiere de un método permanente de trabajo al interior del país, además de la consolidación de un cuerpo profesional que incluye al sector Defensa y al no gubernamental, con habilidades para jugar roles en el escenario internacional, interno (especialmente magallánico) y regional. Este esquema debe funcionar de manera tan eficiente como parecen hacerlo las instituciones de algunos países que mantienen un programa nacional antártico, integrador y con enfoques de mediano plazo.

Para estos efectos, el Consejo de Política Antártica ${ }^{19}$, concebido como órgano rector en todos los planos de acción nacional, está imbuido de una responsabilidad de alta exigencia, que a nuestro juicio no puede cumplir con la celeridad y profundidad necesarias, dadas sus características y la baja frecuencia de sus sesiones, con la posible justificación de la falta de urgencia de los temas que aborda.

Históricamente la Antártica significó un decidido proceso de afirmación de derechos, de posicionamiento ante terceros países, particularmente el Reino Unido, Argentina, y Estados Unidos, y de diseño de algunas estrategias específicas ante el contexto mundial y regional, particularmente desde el siglo XX. Puga Borne y Hunneus ${ }^{20}$ definieron en su momento una prioridad en cuanto a la concreción de la soberanía teniendo en cuenta la unidad geográfica de los territorios de Chile y la Antártica, continua y demostrada por la ciencia y «perceptible a los sentidos», y la cercanía con el continente, más estrecha -señalaban- que a la de cualquier otro país.

\section{Históricamente la Antártica significó un proceso de afirmación de derechos.}

En consecuencia, la política antártica chilena ha sido el resultado de una combinación de las posiciones territoriales tradicionales ${ }^{21}$, de la acción organizadora del Estado $^{22}$, de la participación de Chile en

19 El Consejo de Política Antártica fue creado por el DFL 161, Estatuto Orgánico del Ministerio de Relaciones Exteriores, de 1978, Artículo 13. D. Oficial No 31 de marzo de 1978. Su Reglamento fue adoptado en 1998, D. S. No 495, de Relaciones Exteriores, D. Of. N ${ }^{\circ} 10$ de julio de 1998.

20 Hunneus Gana, A., Antártida, Santiago, 1948, p. 18.

21 Exposición ante el Senado del Ministro de Relaciones Exteriores, Raúl Julliet Gómez, 21 de enero de 1947, Memoria del Ministerio de Relaciones Exteriores, pp. 162-183. La obra de Óscar Pinochet de la Barra, La Antártica Chilena, 2a.ed., Santiago, Editorial Andrés Bello, 1976, es fundamental para el conocimiento de esta época.

22 Berguño, J., Cincuenta Años de Política Antártica, op. cit., pp. 23-34. 
el Tratado Antártico de $1959^{23}$, y de búsqueda de un acomodo jurídico y práctico con las posturas e intereses de terceros países, al amparo del Tratado una vez vigente.

\section{La diplomacia y la experiencia académica han sido factores centrales en la estructuración de la política antártica.}

La diplomacia y la experiencia académica $^{24}$ han sido factores centrales en la estructuración de esta política internacional. Asimismo, en este proceso, las vinculaciones de Chile en los asuntos antárticos tanto a nivel interno como internacional han tenido siempre en cuenta los temas relativos a los recursos naturales, la protección ambiental y la puesta en valor de la proximidad geográfica con los territorios antárticos. El Sistema Antártico y el aprovechamiento de los recursos naturales han sido precisamente uno de los rasgos ordenadores de la política chilena en este contexto más global $^{25}$. En 1979, Zegers postulaba al respecto que para el tratamiento de los recursos antárticos no podría ignorarse el carácter singular del ecosistema antártico, su relación con otros ecosistemas, las realidades jurídicas y políticas del Sistema Antártico y la existencia de reclamaciones soberanas y la larga presencia antártica de diversos países ${ }^{26}$.

Desde los años setenta las iniciativas para regular la conservación y explotación de los recursos naturales vivos y el establecimiento de un régimen para los minerales antárticos marcaron muy fuertemente otra orientación en cuanto al sentido de la participación de Chile y capturaron la atención de la comunidad política nacional, como no se daba desde antes de que se negociara el Tratado Antártico. Es así como se manifestaron demandas internas y posturas diplomáticas complejas cuando se acercaba el momento en que el propio Tratado Antártico podía ser objeto de una solicitud de revisión (1991) y coincidía la discusión sobre el futuro de este tratado con el énfasis en la protección del medio ambiente a nivel internacional y la transición política interna a fines de la década de los ochenta y comienzos de los años noventa ${ }^{27}$.

23 Ver Nota No 7756 de 14 de mayo de 1958, del Ministro de Relaciones Exteriores de Chile al Encargado de Negocios de Estados Unidos en respuesta a la invitación a una conferencia sobre la Antártica. Memoria del Ministerio de Relaciones Exteriores, 1958, pp. 664-666.

24 Las obras de Orrego Vicuña, F. (ed.), La Antártica y sus Recursos, Santiago: Editorial Universitaria, 1983 y Orrego Vicuña, F. y Salinas, A. (eds.), El Desarrollo de la Antártica, Santiago, Editorial Universitaria, 1977, son ejemplos en la materia y constituyen hitos en el estudio de la política antártica chilena. También, Orrego Vicuña, F., M.T. Infante y P. Armanet, op. cit.

25 Zegers, F., «El sistema antártico y la cuestión del aprovechamiento de los recursos en el área», Estudios Internacionales, XII, 1979, № 47, pp. 293-321.

26 Ibid., p. 320.

27 Infante Caffi, M. T., Una Política Antártica para 1991 y el Porvenir, Santiago, Consejo Chileno para las Relaciones Internacionales, Serie Informes Especiales, 1999. 


\section{La política antártica ha estado estrechamente vinculada con las necesidades y objetivos de la política exterior de Chile.}

En el largo plazo, no obstante, la política antártica ha permanecido estrechamente unida a los requerimientos y objetivos de la política exterior por encima de los patrones cambiantes de la política interna de Chile -incluso después de la crisis democrática de los años setenta y la gradual transición hacia la democracia desde fines de los ochenta- cuando se termina la negociación sobre los minerales antárticos. En general, las orientaciones de la política no han experimentado cambios dramáticos derivados de las tendencias ideológicas de los gobiernos en el poder, si bien la negociación relativa a un régimen para los minerales antárticos generó un creciente debate jurídico y político entre los años 1981 y 1988, tal vez el período más activo en torno a la Antártica de toda la historia posterior al Tratado. Por una parte, se encontraban quienes veían en esta negociación una derogación de las normas constitucionales aplicables en Chile a los recursos minerales y que dan sustento a su naturaleza jurídica en el orden interno, y por la otra, no se podía ignorar el impacto de tendencias ambientalistas proyectadas por agrupaciones no gubernamentales que objetaban esta actividad económica en Antártica y desple- garon un intenso lobby en Chile hacia fines de los años ochenta y comienzos de la década siguiente ${ }^{28}$.

No se puede ignorar el impacto del surgimiento de estos nuevos temas en el contexto del Sistema del Tratado Antártico (STA), especialmente en relación con la exploración y explotación de los recursos naturales, el aumento del número de las partes participantes en el Tratado Antártico y el debate en las Naciones Unidas en torno a su legitimidad, entre otros elementos. Sin embargo, en este nuevo siglo los puntos centrales del debate nacional se han situado más bien en torno a la pertinencia y calidad de la investigación científica, el fortalecimiento de una dirección superior de los asuntos antárticos y el papel de la institucionalidad vigente en del Consejo de Política Antártica y los organismos que ejecutan acciones en el continente. Además, se ha centrado la atención en la relación con las potencias antárticas, en especial las que reclaman de soberanía, los países latinoamericanos y la interacción armónica de la Antártica con las visiones de desarrollo magallánico y su proyección sustentable a nivel internacional.

En el plano interno, el Consejo de Política Antártica -cuyo Estatuto Orgánico data de 1978 y su Reglamento de 1998ha propuesto un Plan Estratégico ${ }^{29}$ que contribuye a posicionar de forma destacada los asuntos antárticos y a ampliar en discusión a su respecto. Aun así, el tema antártico continúa perteneciendo mayor-

28 Este interés se reflejó en las Jornadas Antárticas y del Medio Ambiente que se llevaron a cabo en Punta Arenas en ese período.

29 Aprobado el 11 de enero de 2006 por el Consejo de Política Antártica. 
mente a los dominios de la política gubernamental global relacionada con la acción externa del Estado, más que a esferas especializadas en el ámbito de las instituciones internas.

En sus rasgos esenciales, la política de los años ochenta estuvo marcada por la atención de respuesta a la presión de algunos países en favor de la introducción de transformaciones, algunas de orden radical, desde la perspectiva de actores estatales y no estatales que postulaban la aplicación del régimen de patrimonio común de la humanidad, o la crítica a la naturaleza y eficacia del Tratado Antártico y del sistema derivado de él.

En ese período se debatían a nivel político y académico ideas tales como la internacionalización, guiada por el concepto de patrimonio común o la preferencia por un parque natural sin actividad económica alguna como alternativas al establecimiento de un régimen para los recursos minerales que finalmente no se puso en vigor ${ }^{30}$. En cambio, el debate se sitúa más bien en torno al aporte de la actividad antártica al conocimiento de los fenómenos globales y las interacciones con el resto del planeta, la capacidad para conjugar intereses específicos internos del país en su relación con el quehacer antártico general y específico por sectores, y la proyección de las actividades generales desde el extremo sur de América, en un ambiente que es a la vez cooperativo y competitivo.

Con el Protocolo de Madrid de 1991 y el acuerdo para la prohibición de la exploración y explotación de minerales antárticos, entre otros alcances, las señales que envía el Sistema Antártico a la Política nacional implican una orientación más exigente para la acción nacional, lo que se recoge entre otras medida, en las definiciones que el Instituto Antártico Chileno (INACH) ha planteado en su Plan Quinquenal ${ }^{31}$.

\section{El acuerdo para la prohibición de la exploración y explotación de minerales antárticos implica una orientación exigente para la acción nacional.}

En materia científica esto significa no solamente adoptar definiciones conceptuales sino también otros elementos claves, como la promoción de actividades que impliquen una presencia selectiva y útil para dicho propósito y que garantice la ejecución de actividades que otorguen un mayor conocimiento del territorio antártico, tanto de la parte en que se superponen las reclamaciones argentina y británica, como de aquella en que no hay superposición alguna. El Gobierno -a través de las actas del Consejo de Política

30 Política Antártica de Chile, op. cit.; también en Berguño, J., «The Antarctic Park: the issue of environmental protection», The Antarctic Environment and International Law, J. Verhoeven, Ph. Sands \& M. Bruce, (eds.). Londres, Graham \& Trotman, 1992, pp. 103-107. Infante, M.T., «La cuestión antártica», ¿Hacia unas relaciones internacionales de mercado?, Anuario de Políticas Exteriores Latinoamericanas 19901991, PROSPEL, Editorial Nueva Sociedad, Caracas, 1991, pp. 412-423.

$31<$ www.inach.cl>. 
Antártica- ha dado a entender que esta tarea requiere de una mayor participación y coordinación interinstitucional. Es claramente el primer desafío de la actual política antártica, y la capacidad para conducir y coordinar la investigación en esa área recae en el Instituto Antártico Chileno (INACH), creado en 1963 (Ley $\mathrm{N}^{\circ}$ 15.266), dependiente del Ministerio de Relaciones Exteriores.

En esta perspectiva, esta política tiene que dar curso a líneas de trabajo que busquen a la vez privilegiar las conexiones efectivas entre los instrumentos que el Estado ha creado para potenciar las actividades científicas, especialmente con la Comisión Nacional de Ciencia y Tecnología (CONICYT), y promover acuerdos de cooperación con significado real en las investigaciones polares, que impliquen un aprovechamiento sustantivo de las instalaciones, bases y equipamientos en Antártica.

A nuestro juicio, en esta área, las diversas vías para acreditar la actividad científica, tanto que se ejecuta con investigadores y centros académicos nacionales de calidad como la asociada con el ambiente externo en redes internacionales consolidadas, que prestan además utilidad como medios de prestigio o de proyección política, ofrecen una oportunidad especial para nuestro país. El Año Polar Internacional 2007-2008 sirve de marco ordenador objetivo para este tema, pero de todas formas subsiste el desafío de que el país aborde estos temas en una perspectiva or- ganizada y que exija excelencia no solo a los proyectos de investigación con o sin componentes internacionales, sino además. a la capacidad de gestión interinstitucional.

\section{Las vías para acreditar la actividad científica en la Antártica constituyen una oportunidad especial para Chile.}

El INACH está proyectando esta lógica al definir mediante un convenio de colaboración con la CONICYT la realización de un Proyecto de Anillos de Investigación en Ciencia Antártica ${ }^{32}$ en el marco del Programa Bicentenario de Ciencia y Tecnología, que tiene un fuerte acento asociativo. Esta concepción debe dotarse de suficiente energía como para superar la tendencia a la dispersión de esfuerzos entre las instituciones con presencia antártica, así como con la competencia entre actores -especialmente no institucionales- por los mismos fondos para realizar actividades en el continente, y a la diversidad de reglas del juego. Esto se plantea tanto para los laboratorios instalados en la Antártica como para el uso de la infraestructura de transporte y el trabajo a bordo de buques.

Esta materia pone de relieve la cuestión presupuestaria antártica, que al igual que otros sectores del país está sujeta a reglas y metas fiscalizadas externamente. En la práctica, la política antártica debe conciliar dos grandes áreas: por una parte

32 Entre los temas que contiene este proyecto se encuentra la capacitación de investigadores de formación reciente y la formación de estudiantes de nivel de postgrado, así como el impacto antrópico en la Antártica, la diversidad biológica y la biotecnología. 
la proyección a mediano plazo de las líneas de trabajo sumadas a una presencia antártica apta para ese propósito como sustento real del Programa Antártico Nacional que anualmente se realiza, y por la otra, la innovación de los métodos tradicionales de elaboración y distribución del presupuesto antártico y el papel del Consejo de Política Antártica ${ }^{33}$ en la materia, especialmente la asignación de recursos para el funcionamiento de las instituciones que individualmente cumplen labores en Antártica y su rol en la realización de actividades de apoyo a la actividad científica.

\section{El convenio para la realización de un}

Proyecto de Anillos de Investigación en

Ciencia Antártica tiene un fuerte acento asociativo.

\section{OTRAS PROYECCIONES. \\ NUEVAS FRONTERAS}

La proyección de la política antártica como elemento de la acción externa del Estado, y las cuestiones relativas a los recursos marinos, el derecho del mar y el medio ambiente, sitúan otro marco de análisis.

En este ámbito, cinco grandes áreas de trabajo parecen de interés prioritario para una visión amplia y un desarrollo multisectorial:
$\S \quad$ Los temas globales y el aporte de la Antártica al conocimiento de los fenómenos que afectan o impactan al planeta, y la implementación del Protocolo al Tratado Antártico sobre el Medio Ambiente, de 1991. El Plan Estratégico Antártico de 2006 impone altas exigencias a este respecto cuando señala que se trabajará para actualizar las definiciones sobre los intereses estratégicos de nuestro país, expresados en prioridades que incorporen un mayor equilibrio entre la presencia territorial, el robustecimiento de la investigación científica, un aporte sustantivo a la protección ambiental y la valoración económica que hoy se obtiene principalmente de la pesca, el turismo y los servicios a la ciencia internacional que accede a la Antártica occidental a través de Chile.

$\S \quad$ Las relaciones entre el derecho del mar y el régimen antártico, que se expresan en la aplicación de convenciones negociadas para el derecho del mar y su relación con las normas antárticas, tema estudiado pero siempre importante de considerar. Entre otros aspectos, se trata de determinar una posición ante la existencia de una plataforma extendida o ampliada, como lo prevé la Convención de las Naciones Unidas sobre el Derecho del Mar de 1982, en su Artículo 76 y su Anexo II, cuando la plataforma continental se extiende naturalmente hasta el borde exterior del margen conti-

33 En los últimos años ha sido de 840.821 .000 , poco más de $1 / 3$ del total. 
nental, más allá de 200 millas. Dos elementos han debido definirse al respecto: por una parte, la posición de los países antárticos reclamantes de soberanía ante el hecho de que una presentación ante al Comisión de la Plataforma Continental puede llevar a que se discuta el fundamento de su reclamación a la luz del Artículo IV del Tratado, aunque la Comisión sea un órgano exclusivamente técnico, y puede en consecuencia suscitarse un punto de división en el seno del Tratado Antártico, y por el otro, que ante la superposición parcial de reclamaciones en la península antártica deberían también buscarse condiciones para evitar la apertura de nuevos debates y conducir el tema hacia un enfoque común.

Nuestro país ha trabajado el enfoque centrado en esos dos objetivos y contribuyó activamente a las consultas que condujo Nueva Zelandia, a fin de contar con una base común ante la inminencia de la primera presentación de un país antártico, Australia. En este sentido, se procuró promover elementos de acomodación que no suscitasen polémicas que afectaran el consenso antártico, a fin de lograr que los Estados que optasen por hacer una presentación a la Comisión no adoptasen acción alguna a ese respecto. Australia, que finalmente incluyó en su presentación una referencia a las circunstancias del área situada al sur de los $60^{\circ}$ de latitud Sur y el estatus jurídico y político especial de la Antártica de acuerdo con las disposiciones del Tratado Antártico, lo que comprende su Artículo IV, e hizo presente que la Antártica poseía áreas de plataforma continental cuya extensión aún tenía que ser definida, hizo uso de esta opción ${ }^{34}$.

En materia de derecho del mar, otra área lleva a conjugar los intereses y derechos como país antártico con la conservación de los recursos y la acción concreta ante la pesca ilegal, no regulada y no informada, y su impacto para la cohesión en el seno de la Convención para la Conservación de los Recursos Marinos Vivos Antárticos, y las medidas que se adoptan conforme a ella.

- La bioprospección, como actividad que presenta un alto interés científico y económico y que pone en juego la virtud del Protocolo de Madrid para conducir la discusión y la actuación de los Estados en una materia que afecta ciertamente al ecosistema antártico, la libertad de acceso a la información científica que el Tratado Antártico garantiza en su Artículo 3, al mismo tiempo que las reglas de protección de la propiedad intelectual vinculada a la generación de conocimientos y el dominio de técnicas aplicadas al material genético, a partir de su cosecha o recolección. Además, en esta materia la política antártica debe tener en cuenta la activación de la preocupación por la conservación y el uso

34 La alternativa era hacer una presentación parcial, enfoque permitido por el Reglamento de la CLPC. Declaración australiana en Note No 89/2004. Ver en < un.org/Depts/los/clcs_new/submissions_files/ aus04/Documents/aus_doc_es_web_delivery.pdf $>$, y Attachment. 
de la diversidad biológica marina fuera de las zonas de jurisdicción nacional que se ha iniciado en el marco de las Naciones Unidas ${ }^{35}$, y que si bien se referiría a los recursos en la zona no debería tener connotaciones que debiliten el régimen antártico y los intereses de nuestro país.

- La estrecha relación entre el desarrollo austral y la conexión antártica, tanto desde el punto de vista geográfico como en cuanto a la prevención y reglamentación de los impactos de las acciones del hombre. El turismo es ciertamente una de las áreas en que se aprecia un déficit de la política antártica. Ello por cuanto no se ha alcanzado un nivel de desarrollo del proceso decisional que ordene y conjugue claramente las obligaciones derivadas de la participación en el Tratado Antártico y el Sistema derivado, $\mathrm{y}$ las normas internas que rigen al turismo sin distinciones. Hasta ahora, por ejemplo, no se ha podido completar un marco regulatorio razonable para avanzar en materia de turismo, una actividad que pública y ampliamente se realiza en Antártica ${ }^{36}$ y respecto de la cual Chile juega un papel como Estado del puerto o como Estado de origen de las actividades.

- En esta materia, tampoco puede dejar de atenderse a la necesidad de desarrollar el régimen de responsabili- dad por daño al medio ambiente antártico, que prevé el Artículo 16 del Protocolo de Madrid y que ha originado una primera aproximación mediante el Anexo VI. Chile tiene que estudiar su pronta adopción.

Estos elementos y los temas centrales que ha expuesto la política antártica actual no dejan de plantear áreas de interés para el derecho internacional, como son la participación en un régimen internacional que tiende a privilegiar un enfoque cooperativo y práctico respecto de los usos del continente y sus espacios marítimos, y sus consecuencias para la jurisdicción del Estado. Este ha sido uno de los temas centrales de la discusión sobre un régimen en materia de responsabilidad por daño al medio ambiente antártico, cuya primera etapa se ha cumplido con un nuevo Anexo VI sobre Responsabilidad derivada de emergencias medioambientales, aprobado en 2005, y sobre el cual habrá que definir el curso legal mediante el cual Chile será parte.

El objeto principal de este Anexo es establecer obligaciones para que los operadores ${ }^{37}$ antárticos adopten medidas preventivas, planes de emergencia y acciones de respuesta ante emergencias ambientales, así como mecanismos para determinar la responsabilidad a que dará lugar la falta de adopción de tales acciones. Con este Anexo no se agota el tema de la 
responsabilidad por daño al medio ambiente antártico y los ecosistemas dependientes y asociados a que se refiere el Artículo 16 del Protocolo al Tratado Antártico sobre Protección del Medio Ambiente, y la posición chilena ha sido invariablemente que se deberá mantener una posición activa como Parte Consultiva a fin de que este tema continúe estudiándose.

Esta última reflexión lleva a proponer como tema de estudio para este período preparatorio del Año Polar dos ámbitos sobre la cuestión institucional chilena, a partir de las realidades existentes y el papel de los organismos a través de los cuales se da la participación de Chile en el Tratado Antártico, aprovechando integralmente de las capacidades nacionales y regionales en la gestión de los recursos intelectuales, políticos y económicos

Como expresión concreta de este esfuerzo de actualización, una tarea prioritaria debería ser la puesta al día del Estatuto del Territorio Antártico Chileno (DF Ley $\mathrm{N}^{\circ} 298$, de 1956) y de la Ley $\mathrm{N}^{\circ} 11.846$ que le sirve de base. Tareas no faltan en la actual etapa de la política antártica, y el Consejo de Política Antártica debe ponerse en marcha a este respecto. 